\title{
THE SELECTIVE ROLE OF NITRITE IN THE PAO/GAO COMPETITION
}

\author{
Carlota Tayà, Vijay Kumar Garlapati, Albert Guisasola* and Juan A. Baeza
}

*Corresponding author:

Albert Guisasola

Departament d'Enginyeria Química. Escola d'Enginyeria.

Universitat Autònoma de Barcelona, 08193, Bellaterra (Barcelona).Spain.

Tel: 34935811879

email: albert.guisasola@uab.cat

Carlota Tayà

Departament d'Enginyeria Química. Escola d'Enginyeria.

Universitat Autònoma de Barcelona, 08193, Bellaterra (Barcelona).Spain.

Tel: 34935814795

email: carlota.taya@uab.cat

Vijay Kumar Garlapati

Departament d'Enginyeria Química. Escola d'Enginyeria.

Universitat Autònoma de Barcelona, 08193, Bellaterra (Barcelona).Spain. Tel: 34935814798

email: vijaykumar.garlapati@uab.cat

Juan Antonio Baeza

Departament d'Enginyeria Química. Escola d'Enginyeria.

Universitat Autònoma de Barcelona, 08193, Bellaterra (Barcelona).Spain.

Tel: 34935811587

Fax: 34935812013

email: juanantonio.baeza@uab.cat

This is the author's version of a work that was accepted for publication in Chemosphere (Ed. Elsevier). Changes resulting from the publishing process, such as peer review, editing, corrections, structural formatting, and other quality control mechanisms may not be reflected in this document. Changes may have been made to this work since it was submitted for publication. A definitive version was subsequently published in Tayà, $C$. et al. "The selective role of nitrite in the PAO/GAO competition" in Chemosphere, vol. 93, issue 4 (Oct. 2013), p. 612-618. DOI 10.1016/j.chemosphere.2013.06.006 
$>$ Combining EBPR-nitrite pathway with propionate as carbon source selects PAO vs GAO

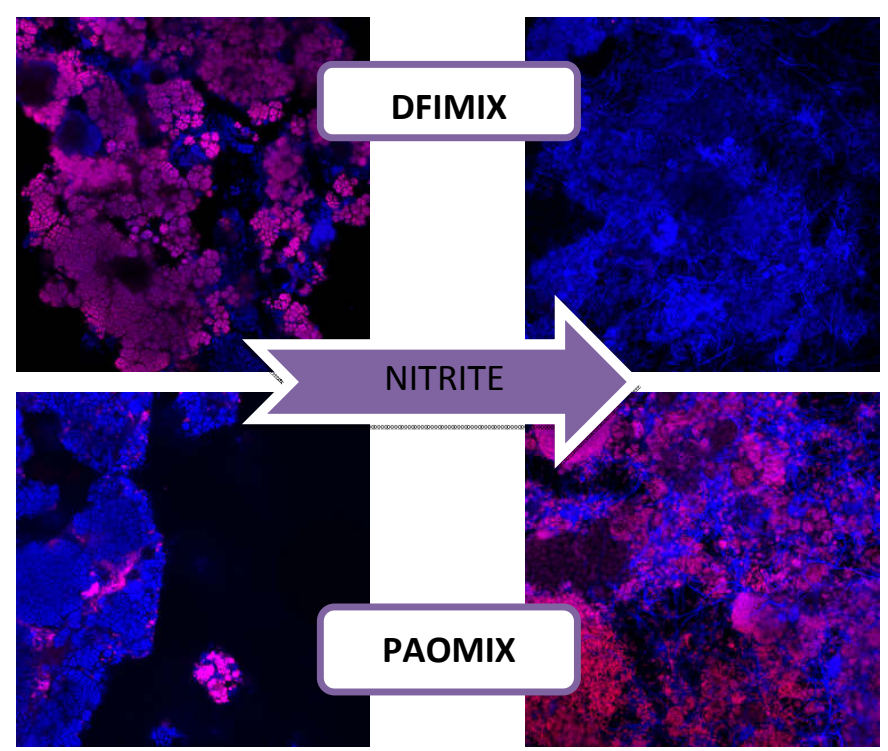


49 ABSTRACT

50 Proliferation of Glycogen Accumulating Organisms (GAO) accounts as one of the 51 major bottlenecks in biological phosphorus removal systems. GAO outcompeting 52 Polyphosphate Accumulating Organisms (PAO) results in lower P-removal. Thus, 53 finding optimal conditions that favour PAO in front of GAO is a current focus of 54 research. This work shows how nitrite can provide a novel strategy for PAO 55 enrichment. A propionate-fed GAO-enriched biomass (70 \% Defluviicoccus I, 18\% Defluviicoccus II and $10 \%$ PAO) was subjected during $50 \mathrm{~d}$ to anaerobic-anoxic

57 conditions with nitrite as electron acceptor. These operational conditions led to a 58 PAO-enriched sludge $(85 \%)$ where GAO were washed out of the system $(<10 \%)$, 59 demonstrating the validity of the new approach for PAO enrichment. In addition, the 60 presented suppression of Defluviicocus GAO with nitrite represents an add-on benefit to 61 the nitrite-based systems since the proliferation of non-desirable GAO can be easily 62 ruled out and added to the other benefits (i.e. lower aeration and COD requirements).

64 KEYWORDS

65 Enhanced biological phosphorus removal (EBPR), nitrite, selection, polyphosphate accumulating organisms (PAO), glycogen accumulating organisms (GAO)

67 


\section{INTRODUCTION}

69

70

71

Nowadays, enhanced biological phosphorus removal (EBPR) has become a widely accepted wastewater treatment technology because of its high sustainability and efficiency. In addition, EBPR is expected to be a viable source of phosphate in the future rather than a technology for biological P removal (Elser and Bennett, 2011). In the EBPR process, biomass is subjected to alternating anaerobic and aerobic/anoxic conditions so that polyphosphate accumulating organisms (PAO) are favoured against other ordinary heterotrophic bacteria. PAO survival capacity relies on their potential to take up organic substrates under anaerobic conditions to synthesize polyhydroxyalkanoates (PHA). The proliferation of several PAO competitors, known as Glycogen Accumulating Organisms (GAO), has become a significant bottleneck in the EBPR process. GAO presence in EBPR systems increases the carbon and chemical requirements, the sludge production and the total overall costs of the plant (Oehmen et al., 2010c; Saunders et al., 2003). Thus, many works (e.g., Oehmen et al., 2010b, López-Vazquez et al., 2009) aim at understanding the competition between PAO and GAO in view of finding the optimal conditions that favours PAO growth against GAO in EBPR systems.

PAO and GAO are not single microorganisms but common terms to describe the observed response of several groups with respect to biological P removal. The latest microbiological studies have revealed the existence of several subgroups of PAO/GAO with different phenotypes (Table 1). Regarding PAO, the Candidatus Accumulibacter phosphatis, hereafter referred as Accumulibacter, are the most widely known PAO in contrast to others as for example Tetrasphera-related or Dechloromonas-related (Oehmen et al., 2010b). Two different types of Accumulibacter (PAOI and PAOII) have been described using the polyphosphate kinase gene as genetic marker (He et al., 2007; 
Peterson et al., 2008) with different denitrification capacity (i.e. PAOI can denitrify nitrate and nitrite whereas PAOII can only use nitrite). Regarding GAO, significant diversity has also been detected, being the Gammaproteobacteria Candidatus Compectibacter phosphatis (from this point on, Competibacter) and the Alfaproteobacteria Defluviicoccus Vanus (from this point on, Defluviicoccus), the most abundant in full-scale plants. The major difference between both groups is their affinity for propionic and acetic acids. Defluviicoccus can be divided into four different clusters (from DFI to DFIV), which have different denitrification capabilities. The denitrification capacities of Defluviicoccus DFI and DFII are shown in Table 1, while capacities of DFIII and DFIV, which are only found in some Waste Water Treatment Plants (WWTP) (Mcilroy and Seviour, 2009), have not been studied yet to the best of our knowledge.

The response of the microorganisms involved in bio-P removal to different electron acceptors and donors (Table 1) is the starting point of the present work. According to this information, none of the reported groups of GAO could survive in an EBPR system with propionate as sole electron donor and nitrite as sole electron acceptor. Hence, these two simultaneous conditions should lead to the washout of the GAO typically found in WWTP and thus, to PAO-enriched sludge. Moreover, as nitrite is a common electron acceptor for PAOI and PAOII, the distribution of these two organisms in the sludge should be similar.

On the other hand, biological nitrogen removal and EBPR are nowadays integrated in activated sludge systems aiming at simultaneous nutrient removal. In these systems, the denitrification capacity of PAO gains a lot of relevance since PAO can reduce the nitrite/nitrate produced in the aerobic phase using the PHA stored in the anaerobic phase. $\mathrm{N}$ removal via the nitrite pathway, i.e. nitritation followed by denitritation, $\mathrm{NH}_{4}{ }^{+}$ 
$\rightarrow \mathrm{NO}_{2}{ }^{-} \rightarrow \mathrm{N}_{2}$ is a more cost effective alternative when treating low COD/N wastewaters due to the lower aeration and COD requirements (Turk and Mavinic, 1987). If the nitrite pathway was implemented, the role of nitrite would be very significant as well as its effect on the PAO/GAO competition. The implementation of the nitrite pathway and EBPR (nitritation and denitritation linked to phosphorus removal) has been reported both in suspended (Marcelino et al., 2011; Zeng et al., 2011) and particularly in granular systems (de Kreuk et al., 2005; Mosquera-Corral et al., 2005; Yilmaz et al., 2008). Recently, Bassin et al. (2012) demonstrated that nitritebased dephosphatation was the main pathway for achieving simultaneous $\mathrm{P}$ and $\mathrm{N}$ removal despite the GAO presence. They detected high PAOII proliferation and the role of GAO was to reduce the nitrate to nitrite.

The aim of this work is to demonstrate that an EBPR sludge operating with propionic acid and nitrite as a sole electron donor and acceptor, respectively, leads to the suppression of GAO activity and to the proliferation of PAO. For this purpose, a highly GAO-enriched sludge was operated under anaerobic/anoxic-nitrite configuration and with propionic acid as a sole carbon source for more than $50 \mathrm{~d}$. The results would help not only for a better understanding of the underlying mechanisms of the PAO/GAO competition but also to understand the role of nitrite in biological nutrient removal systems.

\section{MATERIALS AND METHODS}

\subsection{Experimental set-up}

The experimental set-up consisted of a lab-scale Sequencing Batch Reactor $(\mathrm{SBR})(\mathrm{V}=$ $10 \mathrm{~L}$ ) with oxygen, $\mathrm{pH}$, ORP and temperature probes with a PLC (Siemens S7-226) on top of the control system. The SBR was initially operated for $90 \mathrm{~d}$ under anaerobic- 
aerobic conditions with low influent $\mathrm{P}$ and high $\mathrm{T}$ to favour GAO growth. The cycle length was $6 \mathrm{~h}$ with the following sequence: anaerobic $2 \mathrm{~h}$ (initial feeding $5 \mathrm{~min}$ ), aerobic $3.5 \mathrm{~h}$, sedimentation $25 \mathrm{~min}$ and supernatant extraction $5 \mathrm{~min}(5 \mathrm{~L})$. The synthetic wastewater used in the GAO-enrichment period consisted of the nutrient wastewater solution (4.96 L) (in $\left.\mathrm{g} \cdot \mathrm{L}^{-1}\right)$ : $0.1 \mathrm{NH}_{4} \mathrm{Cl}, 0.044 \mathrm{MgSO}_{4} \cdot 7 \mathrm{H}_{2} \mathrm{O}, 0.16$ $\mathrm{MgCl}_{2} \cdot 6 \mathrm{H}_{2} \mathrm{O}, 0.042 \mathrm{CaCl}_{2} \cdot 2 \mathrm{H}_{2} \mathrm{O}, 0.03 \mathrm{NaHCO}_{3}, 0.0276 \mathrm{KH}_{2} \mathrm{PO}_{4}, 0.0209 \mathrm{~K}_{2} \mathrm{HPO}_{4}$ and 0.005 allylthiourea to inhibit nitrification and $0.30 \mathrm{~mL}$ of nutrient solution, based on Smolders et al. (1994). The carbon source solution (0.04 L) contained only propionic acid for an initial concentration of $225 \mathrm{mg} \mathrm{L}^{-1}$ of COD. The initial phosphate concentration was set to $5 \mathrm{mg} \mathrm{P} \mathrm{L}^{-1}$, resulting in a COD/P ratio of 45 . The hydraulic retention time (HRT) was $12 \mathrm{~h}$. Sludge retention time (SRT) was kept at $15 \mathrm{~d}$ with periodic wastage at the end of the aerobic phase. The temperature was controlled at $25 \pm$ $1{ }^{\circ} \mathrm{C} . \mathrm{HCl}(1 \mathrm{M})$ and $\mathrm{NaOH}(1 \mathrm{M})$ were used to control the $\mathrm{pH}$ in the reactive phases at $7.50 \pm 0.05$. Nitrogen was bubbled during the anaerobic phase to avoid oxygen surface transfer. Dissolved oxygen (DO) was controlled during the aerobic phase between 3.5 and $4.5 \mathrm{mg} \mathrm{L}^{-1}$ to avoid oxygen limitations with an on/off controller.

During the GAO-washout period, the SBR configuration was moved to $2 \mathrm{~h}$ anaerobic, $3.5 \mathrm{~h}$ anoxic, $25 \mathrm{~min}$ of sedimentation and $5 \mathrm{~min}$ for the extraction. Nitrogen was bubbled during all the reactive period. Sodium nitrite was automatically added to the 20. HRT and SRT were kept at the previous values. depletion point in the anoxic phase as defined in a previous work (Vargas et al., 2008). 
This technique consists of the indirect measurement of the proton production (HP) through the monitoring of the amount of base (or acid) dosage necessary to maintain the pH constant (Eq. 1).

$$
\mathrm{HP}=\mathrm{C}_{\mathrm{BASE}} \mathrm{V}_{\mathrm{BASE}}-\mathrm{C}_{\mathrm{ACID}} \mathrm{V}_{\mathrm{ACID}}
$$

where $\mathrm{V}_{\mathrm{BASE}}$ and $\mathrm{V}_{\mathrm{ACID}}$ stand for the accumulated base and acid dosage $(\mathrm{mL})$ and $\mathrm{C}_{\mathrm{BASE}}$ and $\mathrm{C}_{\mathrm{ACID}}$ for base and acid concentration. $\mathrm{Acid}(\mathrm{HCl}$ at $1 \mathrm{M})$ and base $(\mathrm{NaOH}$ at $1 \mathrm{M})$ were added using peristaltic dosage pumps $\left(8 \mathrm{~mL} \cdot \mathrm{min}^{-1}\right)$.

\subsection{Chemical and microbiological analyses}

Phosphorus concentration in $0.22 \mu \mathrm{m}$ filtered samples was measured by a phosphate analyser (115 VAC PHOSPHAX sc, Hach-Lange) based on the Vanadomolybdate yellow method. Nitrite in filtered samples was analysed with Ionic Chromatography (DIONEX ICS-2000). Propionic acid was measured by Agilent Technologies 7820 A GC as described in Guerrero et al. (2012). Glycogen and PHA were measured by triplicate according to the methodology described in Guisasola et al. (2009). Total suspended solids and volatile suspended solids (VSS) were analysed according to APHA (1995).

Fluorescence in situ hybridization (FISH) technique (Amann, 1995) coupled with confocal microscopy was used to quantify the biomass distribution as in Jubany et al. (2009). Hybridizations were performed with Cy3-labelled specific probes (Crocetti et al., 2000; Crocetti et al., 2002) and Cy5-labelled EUBMIX for most bacteria (Daims et al., 1999). Table SM-1 in Supplementary Material (SM) details the probes used in this work.

\subsection{Batch experiment}


192 A batch experiment to test whether PAO could anaerobically store VFA as PHA under limiting poly-P conditions was designed in a $1 \mathrm{~L}$ vessel. This vessel could be operated either under anaerobic/anoxic or aerobic conditions by sparging nitrogen gas or air through a microdiffuser, which ensured good gas transfer to the liquid phase. Gas flow was controlled with a mass flow meter (HiTec 825, Bronckhorst). The $\mathrm{pH}$ (Sentix 81,WTW) and DO (CellOx 325, WTW) probes were connected to its multiparametric terminal (INOLAB 3, WTW) which was in turn connected via RS232 to a PC allowing for data monitoring and storage. The PC also manipulated a high precision microdispenser (Crison Multiburette $2 \mathrm{~S}$ ) to keep the $\mathrm{pH}$ constant at $7.50 \pm 0.01$ with $\mathrm{HCl}(1 \mathrm{M}) / \mathrm{NaOH}(1 \mathrm{M})$ dosage. The reactor was thermally controlled at $25.0 \pm 0.1{ }^{\circ} \mathrm{C}$. The sludge was taken from the SBR at the end of the anoxic phase and placed in the vessel under anaerobic conditions. Two pulses of $100 \mathrm{mg} \mathrm{L}^{-1}$ of propionic acid were added (at 0 and $150 \mathrm{~min}$ ) to exhaust most of internal $\mathrm{P}$ reserves. Then, after a settling period, the supernatant was withdrawn and the biomass was carefully washed to remove the remaining propionic acid and phosphorus in the medium. Next, an aerobic phase was conducted to deplete most of the internally stored PHA. Finally, an anaerobic/nitrite-anoxic cycle was conducted with the addition of $200 \mathrm{mg} \mathrm{L}^{-1}$ of propionic acid. After all $\mathrm{P}$ was released, a settling period to wash the supernatant was carried out to ensure that no propionic acid was present in the next step. Finally, an anoxic phase was performed by adding two pulses of $30 \mathrm{mg} \mathrm{NO}_{2}{ }^{-} \mathrm{N} \mathrm{L}^{-1}$.

\section{RESULTS}

A GAO-enriched EBPR sludge was promoted based on the guidelines proposed by the work of Lopez-Vazquez et al. (2009) under anaerobic/aerobic conditions with propionate as sole carbon source: $\mathrm{COD} / \mathrm{P}$ in the influent was fixed to 45 with very low 
217 initial $\mathrm{P}$ concentration in the reactor $\left(5 \mathrm{mg} \cdot \mathrm{L}^{-1}\right)$. Once the sludge was enriched in GAO,

218 the ratio was moved to a more reasonable value $(\mathrm{COD} / \mathrm{P}=20)$. Figure 1 (LEFT) shows

219 the results obtained from the last of the anaerobic-aerobic cycles, performed with the

220 new COD/P ratio. Propionate was rapidly depleted and linked to some phosphate

221 release, although the $\mathrm{P} / \mathrm{C}$ ratio obtained was 0.20 indicating a significant presence of

222 GAO in the sludge. Nevertheless, $\mathrm{P}$ depletion was observed during the aerobic phase,

223 showing that despite the low $\mathrm{P} / \mathrm{C}$ ratio, the $\mathrm{PAO}$ content in the sludge was enough to

224 achieve complete aerobic P-uptake due to the low initial concentration of P. The FISH

225 results (Fig. 1 RIGHT) supported these observations, indicating a low percentage of

226 PAO (10\%) and a significant amount of GAO (70\% DFI and 18\% DFII), while

227 Compectibacter GAO were scarcely detected, in accordance to the lack of acetate in the

228 feed.

229 Then, oxygen was replaced for nitrite as sole electron acceptor. Figure $2 \mathrm{a}-2 \mathrm{~d}$ shows

230 some examples of the experimental profiles obtained during the $50 \mathrm{~d}$ of anaerobic-

231 anoxic operation. At first glance, the results indicate a fast adaptation to nitrite without

232 any significant inhibition and a significant increase of the anoxic $\mathrm{P}$ uptake activity. This

233 observation is corroborated with the evolution of the maximum nitrite uptake rate

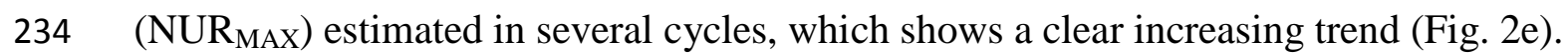

235 Figure 3 shows the evolution of the FISH quantifications during the experimental

236 period. As can be observed, the objective of this work, i.e. proof that nitrite is a strong

237 selection factor in the $\mathrm{PAO} / \mathrm{GAO}$ competition in a propionic-fed environment, was

238 achieved and all the GAO species initially present in the sludge were removed. The

239 percentage of PAO increased in parallel to the GAO depletion. It is also worth

240 mentioning that both PAOI and PAOII were equally favoured in this system. With

241 respect to these FISH measurements, Fig. SM-1 shows selected images for all the 
242 probes tested and provides a better visualisation of the evolution of each biomass

243 population.

244 Finally, Fig. 4 shows a batch cycle conducted at day 41 where the internal storage

245 polymers (glycogen and PHA) were measured. The experimental profiles are in

246 agreement with those found in the literature. Most of the PHA was polyhydroxyvalerate

247 (PHV) $(66 \pm 9 \%)$ in accordance to the use of propionate as sole carbon source (Pijuan

248 et al. 2009). Table 2 compares the experimental ratios obtained with other similar

249 experimental works and with those estimated theoretically with metabolic modelling.

250 As can observed, the experimental values are unexpectedly far from those typical of

251 PAO if one takes into account the high enrichment showed by the FISH images. These

252 results will be discussed in the following section.

\section{DISCUSSION}

255

The major outcome of this work is the demonstration that nitrite can be used as the selection factor in the PAO/GAO competition when Defluviicocus GAO are predominant in the sludge. The operation of an enriched culture of Defluviicocus under anaerobic/anoxic-nitrite conditions with propionate as sole carbon source resulted in the washout of Defluviicocus in parallel to the growth of PAO showing the feasibility to obtain a highly enriched culture of PAO (85\%) even from an enriched culture of Defluviicocus. GAO enrichment was initially forced with an unusually high COD/P and 262 then, this ratio was moved it into a conventional value $(\mathrm{COD} / \mathrm{P}=20)$. While this 263 decrease may have contributed towards the enrichment of PAO over GAO, using this ratio as sole selection factor has not been reported as a successful strategy for obtaining a highly PAO-enriched sludge without GAO. 
The operation with propionate led to the selection of Defluviicocus GAO versus

267 Competibacter GAO, which have been found to be favoured with acetate as carbon source (Oehmen et al., 2005b). Competibacter are able to use nitrite as electron acceptor (Kong et al., 2006b) and hence, the strategy of using acetate as sole carbon source under anaerobic/anoxic-nitrite conditions would not have led to an enriched culture of PAO. The observed Defluviicocus GAO washout is somehow in contrast to the findings of Liu et al. (2010) with a highly-enriched culture of Competibacter GAO obtained using acetate as sole carbon source. They found that nitrite dosage was more adverse for the aerobic PAO metabolism rather than for the aerobic GAO metabolism and suggested that nitrite could provide a competitive advantage of Competibacter over PAO. In our case, Defluviicocus were much more abundant (DFI 70\% and DFII $18 \%$ ) than Competibacter and the anaerobic/anoxic-nitrite conditions were demonstrated to be more favourable for PAO than for Defluviicocus. Based on these results, it seems clear that nitrite-pathway EBPR systems should focus on achieving a carbon source with high propionate fractions over acetate to combine the positive effects of nitrite and propionate on PAO selection. In this sense, Chen et al. (2013) proposed recently a novel strategy to increase the propionate content in the wastewater.

With respect to the utilization of nitrite by Defluviicocus, it was reported that DFI cannot use nitrite as an electron acceptor (Wang et al., 2007) whereas DFII is unable to from the theoretical ones widely accepted for PAO. The explanation for such high values would lay on the low amount of $\mathrm{P}$ in the influent used, which resulted in a low poly-P content of the biomass. Under these conditions, PAO can take up propionate 
using glycogen as primary energy source (a typical GAO behaviour) for survival purposes. The fact that PAO can activate the glycolytic pathway in order to balance the lack of energy derived from low poly-P hydrolysis has already been reported in the literature (Acevedo et al., 2012; Erdal et al., 2008; Zhou et al., 2008). This ability of PAO to store volatile fatty acid (VFA) as PHA anaerobically under limiting poly-P conditions and use it afterwards was observed in a batch experiment (Fig. 5). The experiment, conducted at the end of the experimental period, aimed at forcing PAO to take up propionate anaerobically under scarce poly-P conditions. For this aim, most of the internal poly-P was depleted under anaerobic conditions with an excess of carbon addition, showing a $\mathrm{P} / \mathrm{C}$ ratio of 0.21 . Then, the medium was replaced for a phosphatefree medium and the system was left overnight under aerobic conditions. Propionate was added again under anaerobic conditions and P-release was very low despite the high amount of COD taken up (P/C $<0.01)$, indicating that PAO (85\% of PAO in the sludge) had changed their behaviour from PAO to GAO. Finally, the medium was replaced to remove any propionate remaining and nitrite was added. Successful denitritation was observed showing that PAO were responsible for this nitrite reduction since Defluviicocus are not able to reduce nitrite.

Moreover, when dealing with nitrite-based EBPR systems, $\mathrm{pH}$ should be also considered. High pH favours PAO against GAO due to kinetics (Oehmen et al., 2005a), but when nitrite is present in the system it has an additional positive effect because it results in less free nitrous acid (FNA) concentration, a reported P-uptake inhibitor (Pijuan et al., 2010). Hence, the combination of nitritation with PAO denitritation should be carefully performed to avoid high FNA concentration that would be detrimental for EBPR stability. In our experimental study, the utilization of a controlled 
$315 \mathrm{pH}$ of 7.5, linked to the careful addition of nitrite in several pulses was probably helpful 316 to avoid the observance of any FNA inhibition.

317 This work also shows how a conventional anaerobic/aerobic EBPR system can be 318 directly adapted to anaerobic/anoxic conditions with nitrite as electron acceptor from 319 day 1 without any intermediate anaerobic/anoxic/aerobic configuration as previously 320 reported (Vargas et al., 2011). The nitrite-based anoxic $\mathrm{P}$ uptake rate was, however, much slower than that under aerobic conditions and hence, nitrite should be wisely dosed to avoid its presence at the end of the anoxic phase. Nitrite entering the anaerobic phase can be very detrimental for the PAO growth (see discussion below). Despite these limitations, the SBR got rapidly acclimatised to nitrite as an electron acceptor and anoxic-P uptake was complete in all the period. The last cycles of the period were very similar (Fig. 2) and typical of an EBPR cycle with complete P removal. P-uptake was usually higher than P-release and net P removal was usually achieved.

The fast adaptation to nitrite is in agreement with the fact that both PAOI and PAOII initially present in the sludge are able to reduce nitrite (Oehmen et al., 2010b), and hence nitrite utilization as sole electron acceptor should not be a selection factor between PAOI and PAOII. This observation was confirmed with the final FISH measurements (65\% PAOI versus $35 \%$ PAOII, Fig. 3) and with a batch experiment with nitrate as electron acceptor (Fig. SM-2). Nitrate utilisation did not need an acclimatisation period at all and complete anoxic $\mathrm{P}$ uptake was achieved. These results contrast with previous works where nitrite-based selections led to systems without nitrate-reducing capabilities (Guisasola et al., 2009; Jiang et al., 2006). In this sense, a complete understanding on the competition between PAOI and PAOII in nitritereducing EBPR environments is still needed. Selecting PAOI in front of PAOII would be more interesting in view of increasing flexibility (i.e. both nitrate and nitrite could be 
treated). Otherwise, a PAOII-enriched sludge would be desirable to integrate partial nitrification and EBPR provided the specific nitrite reduction rate of PAOII seems to be higher than PAOI.

Two different practical implications can be drawn from this work. On the one hand, nitrite and propionate could be used as strategy to enrich a bio-P sludge with PAO. PAO have yet to be isolated and novel strategies for its enrichment are very interesting for fundamental studies on PAO physiology and biochemistry (Lu et al., 2006). On the other hand, the suppression of Defluviicocus GAO when nitrite is the electron acceptor represents an add-on to the nitrite-based EBPR systems since the proliferation of nondesirable GAO can be easily ruled out and added to the other benefits (i.e. lower aeration and COD requirements).

351 Finally, these results provide useful recommendations for improving the EBPR activity in full-scale WWTP. The implementation of nitrite pathway for nitrogen removal and the utilization of external carbon sources fermentable to propionic acid, as glycerol (Guerrero et al., 2012) or food waste (Chen et al., 2013), should favour PAO in front of both Competibacter and Defluviicocus GAO and hence would provide a more stable and efficient $\mathrm{P}$ removal.

\section{CONCLUSIONS}

A bio-P sludge enriched in Defluviicocus GAO (70 \% DFI, 18\% DFII and $10 \%$ PAO) was operated under anaerobic/anoxic-nitrite configuration, achieving the washout of these microorganisms in parallel to the growth of PAO (up to 85\%) and demonstrating that nitrite is a key selection factor in the $\mathrm{PAO} / \mathrm{GAO}$ competition. This novel strategy not only allows the achievement of a highly PAO-enriched activated sludge, but also demonstrates that the integration of denitritation with EBPR favours the 
suppression of Defluviicocus GAO and represents an additional advantage for the EBPR

366 configurations using anoxic-nitrite conditions, in addition to lower aeration and COD

367 requirements.

368

\section{ACNOWLEDGEMENTS}

370 Carlota Tayà acknowledges the support of Universitat Autònoma de Barcelona with a

371 pre-doctoral grant. Vijay Kumar Garlapati is thankful for the postdoctoral research

372 fellowship at UAB inside the Alliance4universities program. This work was supported

373 by the Spanish Ministerio de Economía y Competitividad (CTM2010-20384). The

374 authors are members of the GENOCOV research group (Grup de Recerca Consolidat de

375 la Generalitat de Catalunya, 2009 SGR 815).

376

377 


\section{REFERENCES}

Acevedo, B., Oehmen, A., Carvalho, G., Seco, A., Borrás, L., Barat, R., 2012. Metabolic shift of polyphosphate-accumulating organisms with different levels of polyphosphate storage. Water Res. 46 (6), 1889-1900.

Amann, R., 1995. Fluorescently labled, ribosomal-RNA-targeted oligonucleotide probes in the study of microbial ecology. Mol. Ecol. 4,543-553.

APHA, 1995. Standard Methods For The Examination Of Water And Wastewater. 19th ed. American Publishers Health Association, Washington DC.

Bassin, J.P., Kleerebezem, R., Dezotti, M., van Loosdrecht, M.C.M., 2012. Simultaneous nitrogen and phosphate removal in aerobic granular sludge reactors operated at different temperatures. Water Res. 46(12), 3805-3816.

Chen, Y.G., Liu, Y., Zhou, Q., Gu, G.W., 2005. Enhanced phosphorus biological removal from wastewater - effect of microorganism acclimatization with different ratios of short-chain fatty acids mixture. Biochem. Eng. J. 27, 24-32.

Chen, Y.G., Li, X., Zheng, X., Wang D.B., 2013. Enhancement of propionic acid fraction in volatile fatty acids produced from sludge fermentation by the use of food waste and Propionibacterium acidipropionici. Water Res. 47(2), 615-622.

Crocetti, G.R., Banfield, J.F., Keller, J., Bond, P.L., Blackall, L.L., 2002. Glycogenaccumulating organisms in laboratory-scale and full-scale wastewater treatment processes. Microbiology 148, 3353-3364.

Crocetti, G.R., Hugenholtz, P., Bond, P.L., Schuler, A., Keller, J., Jenkins, D., Linda, L., Keller, R.G., Blackall, LL., 2000. Identification of polyphosphate-accumulating organisms and design of 16S rRNA-directed probes for their detection and quantitation. Appl. Environ. Microbiol. 66(3), 1175-1182. 
402

403

404

405

406

407

408

409

410

411

412

413

414

415

416

417

418

419

420

421

422

423

424

425

Daims, H., Bruhll, A., Amann, R., Schleifer, K., Wagner, M., 1999. The domainspecific probe EUB338 is insufficient for the detection of all bacteria: development and evaluation of a more comprehensive probe set. Systematic Appl. Microbiol. $22,434-444$.

de Kreuk, M.K., Pronk, M., van Loosdrecht, M.C.M., 2005. Formation of aerobic granules and conversion processes in an aerobic granular sludge reactor at moderate and low temperatures. Water Res. 39(18), 4476-4484.

Elser, J., Bennett, E., 2011. Phosphorus cycle : A broken biogeochemical cycle. Nature. 478, 29-31.

Erdal, U.G., Erdal, Z.K., Daigger, G.T., Randall, C.W., 2008. Is it PAO-GAO competition or metabolic shift in EBPR system? Evidence from an experimental study. Water Sci. Technol. 58(6), 1329-1334.

Guerrero, J., Tayà, C., Guisasola, A., Baeza, J.A., 2012. Glycerol as a sole carbon source for enhanced biological phosphorus removal. Water Res. 46(9), 29832991.

Guisasola, A., Qurie, M., Vargas, M.D.M., Casas, C., Baeza, J.A., 2009. Failure of an enriched nitrite-DPAO population to use nitrate as an electron acceptor. Process Biochem. 44(7), 689-695.

He, S., Gall, D.L., McMahon, K.D., 2007. “Candidatus Accumulibacter” population structure in enhanced biological phosphorus removal sludges as revealed by polyphosphate kinase genes. Appl. Environ. Microbiol. 73(18), 5865-5874.

Jiang, Y., Wang, B., Wang, L., Chen, J., He, S., 2006. Dynamic response of denitrifying poly-P accumulating organisms batch culture to increased nitrite concentration as electron acceptor. J. Environ. Sci. Heal. A. 41(11), 2557-2570. 
Jubany, I., Lafuente, J., Carrera, J., Baeza, J.A., 2009. Automated thresholding method (ATM) for biomass fraction determination using FISH and confocal microscopy. J. Chem. Technol. Biotechnol. 84(8), 1140-1145.

Kong, Q.X., Wang, X.W., Jin, M., Shen, Z.Q., Li, J.W., 2006a. Development and application of a novel and effective screening method for aerobic denitrifying bacteria. FEMS Microbiol. Lett. 260, 150-155.

Kong, Y.H., Xia, Y., Nielsen, J.L., Nielsen, P.H., 2006b. Ecophysiology of a group of uncultured gammaproteobacterial glycogen- accumulating organisms in full-scale enhanced biological phosphorus removal wastewater treatment plants. Environ. Microbiol. 8 (3), 479-489.

Liu, Y., Pijuan, M., Yuan, Z., 2010. The effect of free nitrous acid on the anabolic and catabolic processes of glycogen accumulating organisms. Water Res. 44(9), 29012909.

Lopez-Vazquez, C.M., Oehmen, A., Hooijmans, C.M., Brdjanovic, D., Gijzen, H.J., Yuan, Z., van Loosdrecht, M.C.M., 2009. Modeling the PAO - GAO competition : Effects of carbon source, $\mathrm{pH}$ and temperature. Water Res. 43(2), 450-462.

Lu, H., Oehmen, A., Virdis, B., Yuan, Z., 2006. Obtaining highly enriched cultures of Candidatus Accumulibacter phosphates through alternating carbon sources. Water Res. 40(29), 3838-3848.

Marcelino, M., Wallaert, D., Guisasola, A., Baeza, J.A., 2011. A two-sludge system for simultaneous biological C, N and P removal via the nitrite pathway. Water Sci. Technol. 64(5), 1142-1147.

Mcilroy, S., Seviour, R.J., 2009. Elucidating further phylogenetic diversity among the organisms in activated sludge. Environ. Microbiol. 1(6), 563-568. 
Meyer, R.L., Saunders, A.M., Blackall, L.L., 2006. Putative glycogen- accumulating organisms belonging to Alphaproteobacteria identified through rRNA-based stable isotope probing. Microbiology 152, 419-429.

Mosquera-Corral, A., de Kreuk, M.K., Heijnen, J.J., van Loosdrecht, M.C.M., 2005. Effects of oxygen concentration on N-removal in an aerobic granular sludge reactor. Water Res. 39(12), 2676-2686.

Oehmen, A., Vives, T. M., Lu, H., Yuan, Z., and Keller, J., 2005a. The effect of pH on the competition between polyphosphate-accumulating organisms and glycogenaccumulating organisms. Water Res. 39(15), 3727-37.

Oehmen, A., Yuan, Z.G., Blackall, L.L., Keller, J., 2005b. Comparison of acetate and propionate uptake by polyphosphate accumulating organisms and glycogen accumulating organisms. Biotechnol. Bioeng. 91 (2), 162-168.

Oehmen, A., Carvalho, G., Freitas, F., Reis, M. A. M. 2010a. Assessing the abundance and activity of denitrifying polyphosphate accumulating organisms through molecular and chemical techniques. Water Sci. Tecnol. 61(8), 2061-2068.

Oehmen, A., Carvalho, G., Lopez-Vazquez, C. M., van Loosdrecht, M. C. M., Reis, M. A. M. 2010b. Incorporating microbial ecology into the metabolic modelling of polyphosphate accumulating organisms and glycogen accumulating organisms. Water Res. 44(17), 4992-5004.

Oehmen, A., Lopez-Vazquez, C. M., Carvalho, G., Reis, M. A. M., van Loosdrecht, M. C. M., 2010c. Modelling the population dynamics and metabolic diversity of organisms relevant in anaerobic/anoxic/aerobic enhanced biological phosphorus removal processes. Water Res. 44(15), 4473-4486.

Peterson, S.B., Warnecke, F., Madejska, J., Mcmahon, K.D., Hugenholtz, P., 2008. Environmental distribution and population biology of Candidatus Accumulibacter, 
a primary agent of biological phosphorus removal. Environ. Microbiol. 10(10), 2692-2703.

477 Pijuan, M., Casas, C., Baeza, J.A., 2009. Polyhydroxyalkanoate synthesis using different carbon sources by two enhanced biological phosphorus removal microbial communities. Process Biochem. 44(1), 97-105. 6072.

Saunders, A.M., Oehmen, A., Blackall, L.L., Yuan, Z., Keller, J., 2003. The effect of

Pijuan, M., Ye, L., Yuan, Z. 2010. Free nitrous acid inhibition on the aerobic metabolism of poly-phosphate accumulating organisms. Water Res. 44, 6063GAOs (glycogen accumulating organisms) on anaerobic carbon requirements in full-scale Australian EBPR (enhanced biological phosphorus removal) plants. Water Sci. Technol. 47(11), 37-43.

Smolders, G.J.F., van der Meij, J., van Loosdrecht, M.C.M., Heijnen, J.J., 1994. Model of the anaerobic metabolism of the biological phosphorus removal process: stoichiometry and pH influence. Biotechnol. Bioeng. 43(6), 461-470.

Turk, O., Mavinic, D.S., 1987. Benefits of using selective inhibition to remove nitrogen from highly nitrogenous wastes. Environ. Technol. Lett. 8(1-12), 419-426.

Vargas, M., Guisasola, A., Artigues, A., Casas, C., Baeza, J.A., 2011. Comparison of a nitrite-based anaerobic-anoxic EBPR system with propionate or acetate as electron donors. Process Biochem. 46(3), 714-720.

Vargas, M., Guisasola, A., Lafuente, J., Casas, C., Baeza, J.A., 2008. On-line titrimetric monitoring of anaerobic-anoxic EBPR processes. Water Sci. Technol. 57(8), 11491154. 
498 Wang, X., Zeng, R.J., Dai, Y., Peng, Y., Yuan, Z., 2007. The denitrification capability

499 of cluster 1 Defluviicoccus vanus-related glycogen-accumulating organisms.

$500 \quad$ Biotechnol. Bioeng. 99(6), 1329-1336.

501 Yilmaz, G., Lemaire, R., Keller, J., Yuan, Z., 2008. Simultaneous nitrification,

502 denitrification and phosphorus removal from nutrient-rich industrial wastewater

503 using granular sludge. Biotechnol. Bioeng. 100(3), 529-541.

504 Zeng, W., Yang, Y., Li, L., Wang, X., Peng, Y., 2011. Effect of nitrite from nitritation

505 on biological phosphorus removal in a sequencing batch reactor treating domestic

506 wastewater. Bioresource Technol. 102(12), 6657-6664.

507 Zhou, Y., Pijuan, M., Zeng, R. J., Lu, H., Yuan, Z., 2008. Could polyphosphate-

508 accumulating organisms (PAOs) be glycogen-accumulating organisms (GAOs)? Water

509 Res. 42, 2361-2368.

510

511 
513 Table 1 Comparison of the preferred VFA and the denitrification capabilities for the

514 different PAO/GAO subgroups (adapted from Oehmen et al., 2010b)

\begin{tabular}{|c|c|c|c|}
\hline & \multirow{2}{*}{ Preferred VFA } & \multicolumn{2}{|c|}{ Denitrification capacity } \\
\hline & & $\mathrm{NO}_{3}^{-}$ & $\mathrm{NO}_{2}^{-}$ \\
\hline Accumulibacter PAO I & \multirow{2}{*}{ Acetate and Propionate } & $\checkmark$ & $\checkmark$ \\
\hline Accumulibacter PAO II & & $\mathbf{x}$ & $\checkmark$ \\
\hline Competibacter & \multirow{4}{*}{ Acetate } & & \\
\hline Sub-groups $1,4,5$ & & $\checkmark$ & $x$ \\
\hline Sub-groups 3,7 & & $\boldsymbol{x}$ & $x$ \\
\hline Sub-group 6 & & $\checkmark$ & $\checkmark$ \\
\hline Defluviicoccus DFI & \multirow{2}{*}{ Propionate } & $\checkmark$ & $\boldsymbol{x}$ \\
\hline Defluviicoccus DFII & & $x$ & $\boldsymbol{x}$ \\
\hline
\end{tabular}

515

516 
518 Table 2 Comparison of the typical PAO/GAO ratios of this work with others found in 519 the literature for propionic acid as sole carbon source.

\begin{tabular}{|c|c|c|c|c|}
\cline { 3 - 5 } \multicolumn{2}{l|}{} & $\begin{array}{c}\text { Gly/VFA } \\
\left(\mathrm{mmolC} . \mathrm{mmol}^{-1} \mathrm{C}\right)\end{array}$ & $\begin{array}{c}\text { PHA } \\
\left(\mathrm{mmolC} . \mathrm{mmol}^{-1} \mathrm{C}\right)\end{array}$ & $\begin{array}{c}\text { P/C } \\
\left(\mathrm{mmolP} . \mathrm{mmol}^{-1} \mathrm{C}\right)\end{array}$ \\
\hline Chen et al., 2005 & PAO & & 1.02 & 0.45 \\
\hline Pijuan et al., 2009 & PAO & 0.45 & 0.64 & 0.27 \\
\hline Oehmen et al., & PAO & 0.33 & 1.22 & 0.3 \\
\cline { 2 - 5 } 2010a & GAO & $0.67-1.0$ & $1.50-1.78$ & 0.34 \\
\hline This Study & PAO & 0.49 & 1.47 & \\
\hline
\end{tabular}

520

521

522 
523 Table S1 Summary of the probes used in the FISH measurements

\begin{tabular}{ccc}
\hline $\begin{array}{c}\text { Short } \\
\text { name }\end{array}$ & Specificity & Reference \\
\hline EUBMIX & Most bacteria & Daims et al. 1999 \\
\hline PAOMIX & Accumulibacter phosphatis & Croccetti et al. 2000 \\
\hline PAO I & $\begin{array}{c}\text { Cluster I of Accumulibacter } \\
\text { phosphatis }\end{array}$ & Flowers et al. 2009 \\
\cline { 1 - 2 } PAO II & $\begin{array}{c}\text { Cluster II of Accumulibacter } \\
\text { phosphatis }\end{array}$ & \\
\hline GAOMIX & Competibacter phophatis & Croccetti et al. 2002 \\
\hline DFIMIX & Cluster I of Defluviicoccus vanus & Wong et al. 2004 \\
\hline DFIIMIX & Cluster II of Defluviicoccus vanus & Meyer et al. 2006 \\
\hline DFIII & Cluster III of Defluviicoccus vanus & $\begin{array}{c}\text { Mcilroy and Seviour } \\
(2009)\end{array}$ \\
\hline
\end{tabular}

524

525 

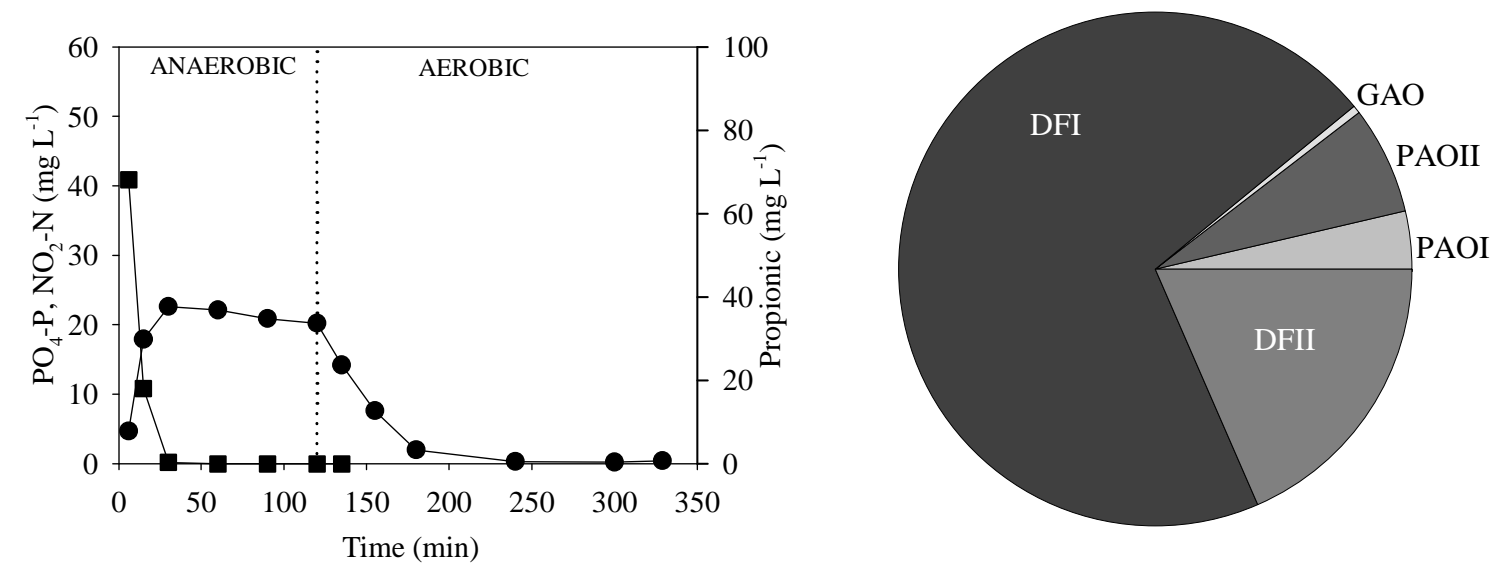

528

529

530 Figure 1 (LEFT) Experimental propionic acid ( $\bullet$ ) and phosphate $(\bullet)$ profiles for the 531 GAO-enriched sludge. (RIGHT) Microbial distribution according to FISH images.

532

533 

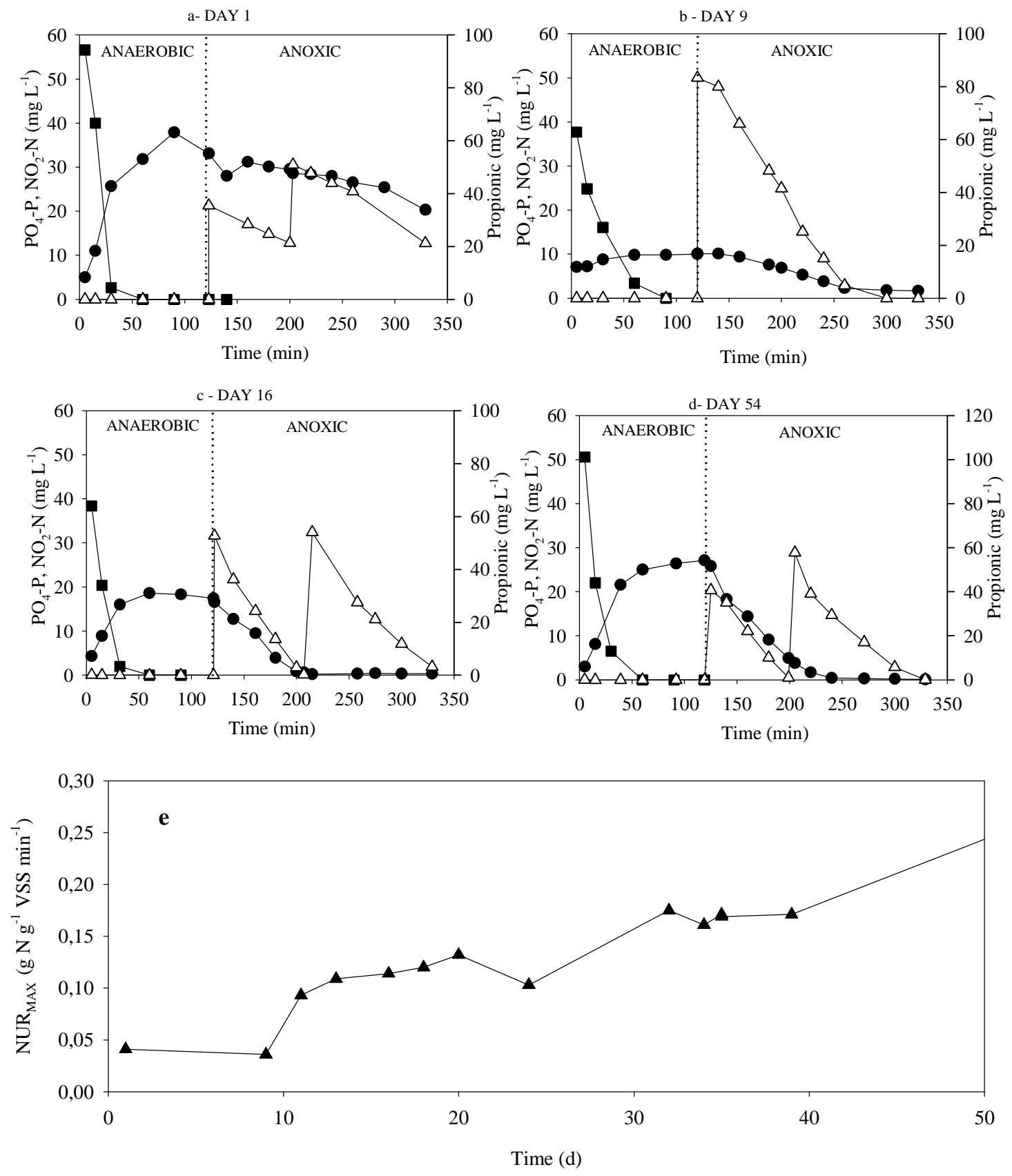

537 Figure 2 (a to d) Experimental propionic acid (घ), phosphate $(\bullet)$ and nitrite $(\triangle)$

538 profiles for some cycles during the experimental period. (e) NUR $\mathrm{MAX}_{\text {evolution during }}$ 539 the experimental period. 


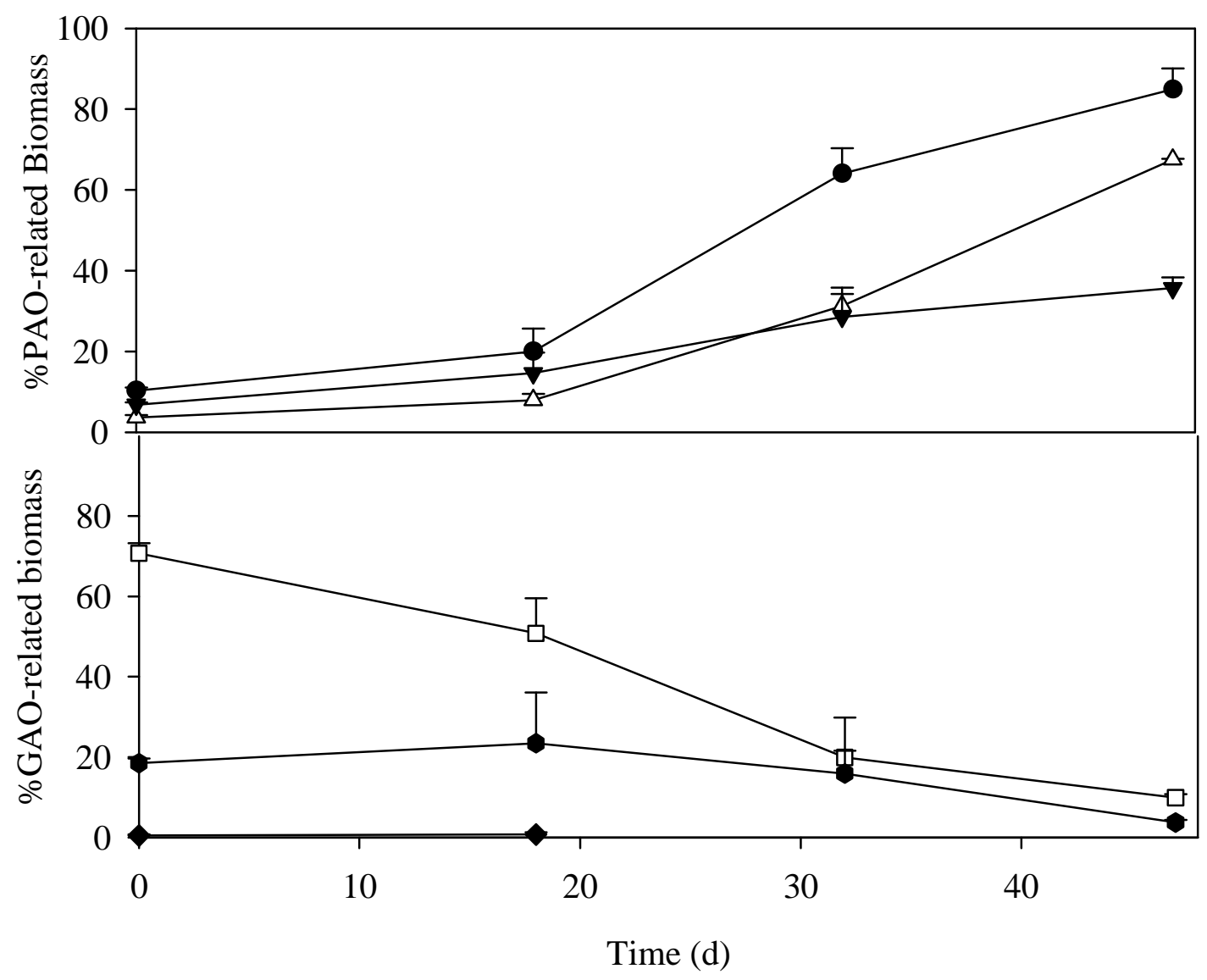

543

544 Figure 3 FISH distribution percentages of the biomass. PAO-related biomass (UP):

545 PAOMIX $(\bullet)$, PAO I $(\triangle)$ and PAO II $(\boldsymbol{\nabla})$. GAO-related biomass (DOWN): GAOMIX

$(\bullet), \operatorname{DF} 1 \mathrm{MIX}(\square)$ and DF2MIX ( $)$. 


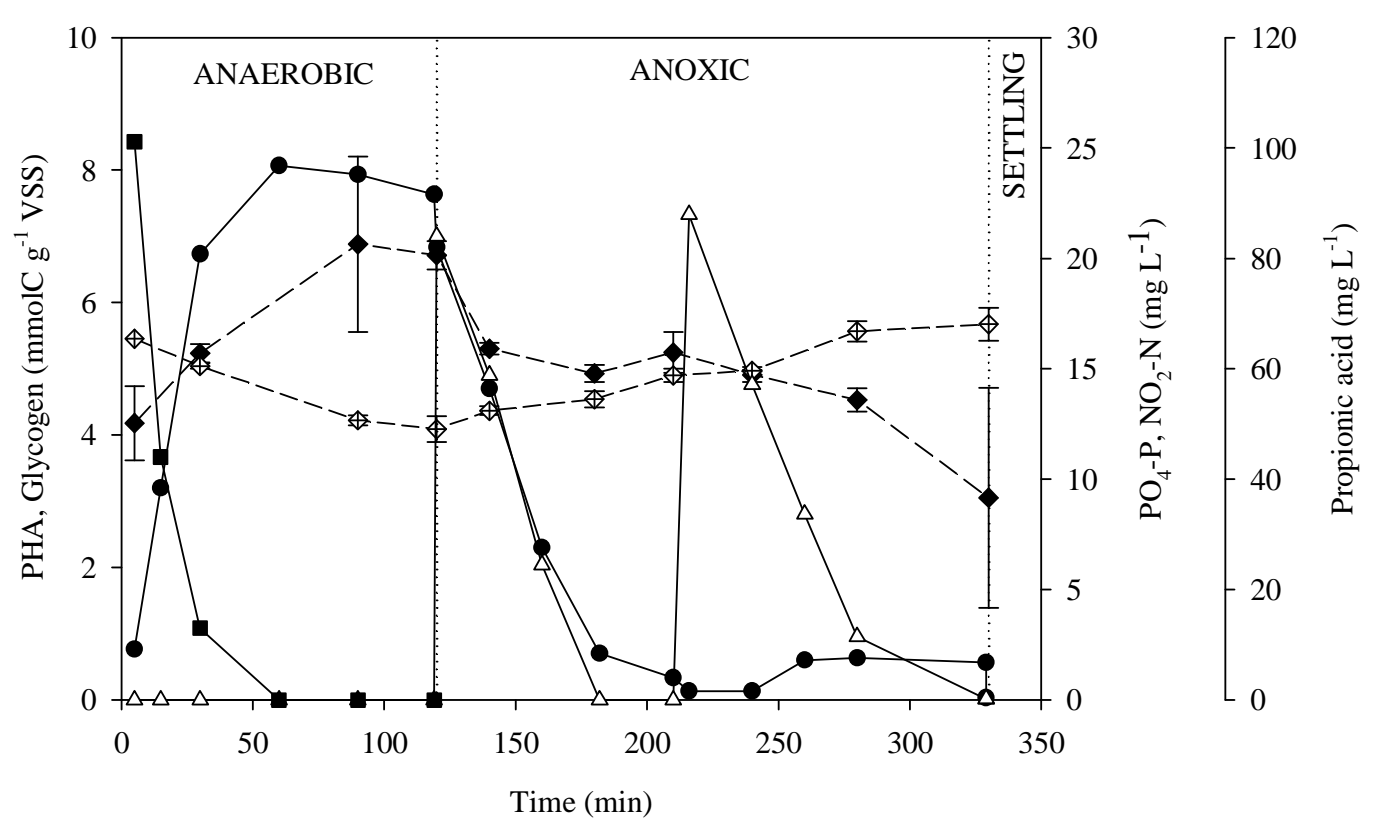

550

551 Figure 4 Experimental PHA $(\diamond)$, glycogen $(\diamond)$, propionic acid $(\bullet)$, phosphate $(\bullet)$ and 552 nitrite $(\triangle)$ profiles for a cycle from day 41 .

553

554 


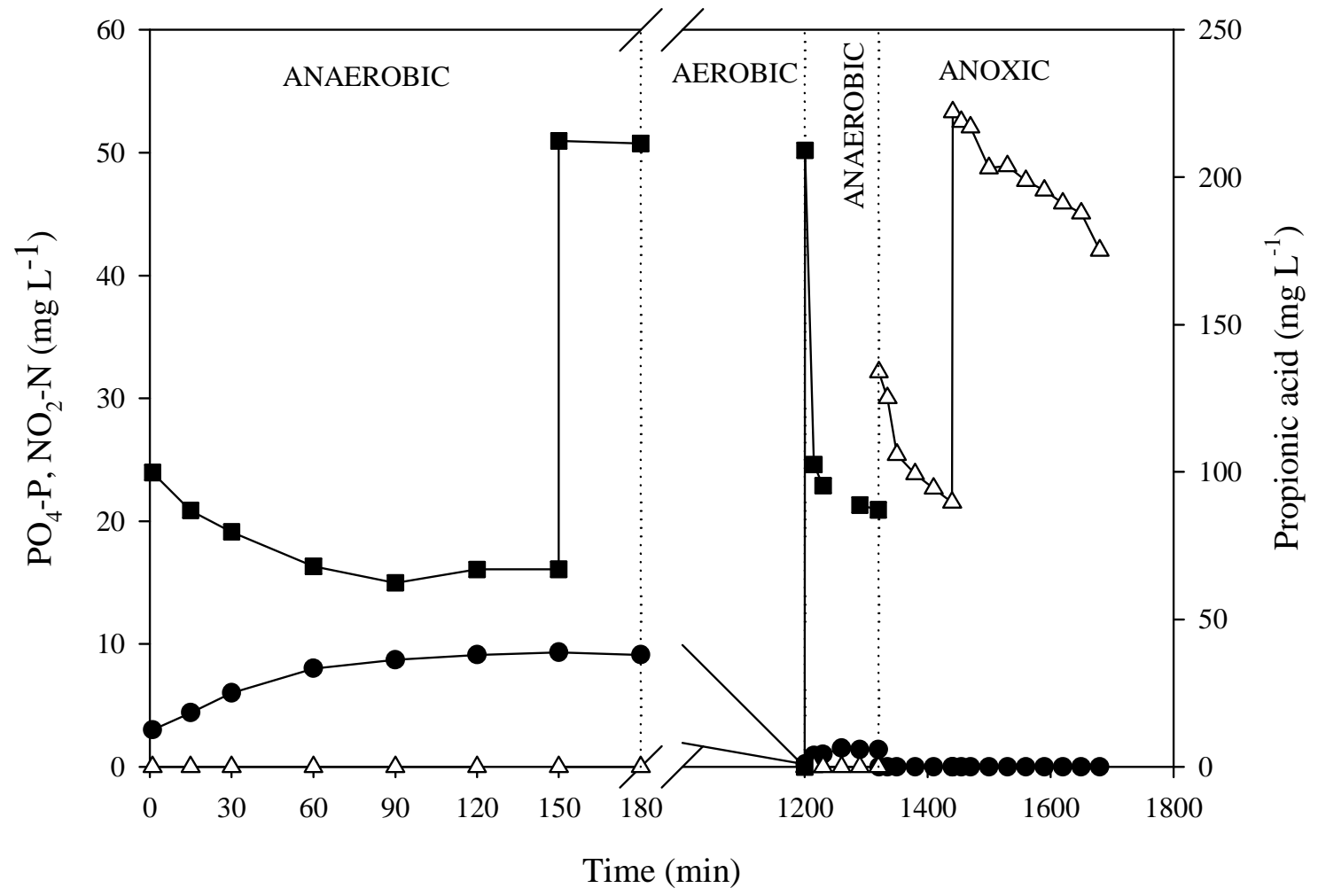

555

556 Figure 5 Experimental propionic acid $(\boldsymbol{\bullet})$, phosphate $(\bullet)$ and nitrite $(\triangle)$ profiles for the 557 batch experiment of PAO behaving like GAO. 

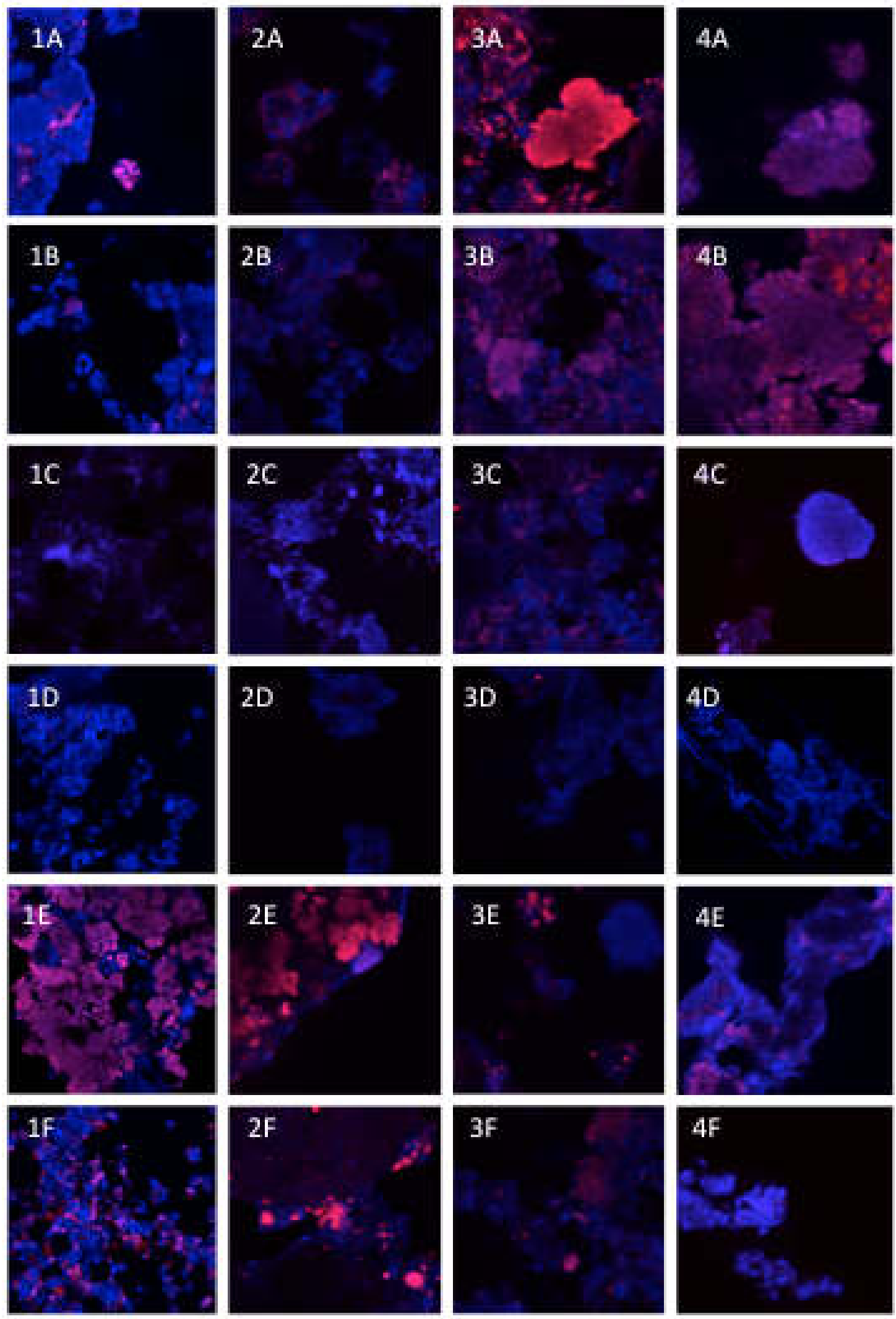

3F

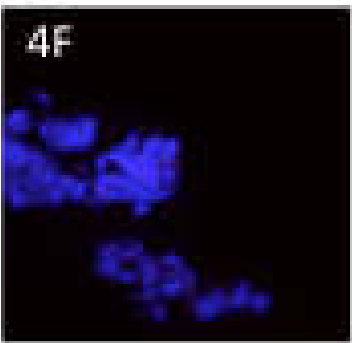


561 Figure S1 FISH/CLSM representative images of the sludge from the SBR reactor

562 during the enrichment. A. PAOMIX, B. PAO clade I, C. PAO clade II, D. GAOMIX, E.

563 DF1MIX, F. DF2MIX. Specific probe is shown in pink and EUBMIX probe in blue. 


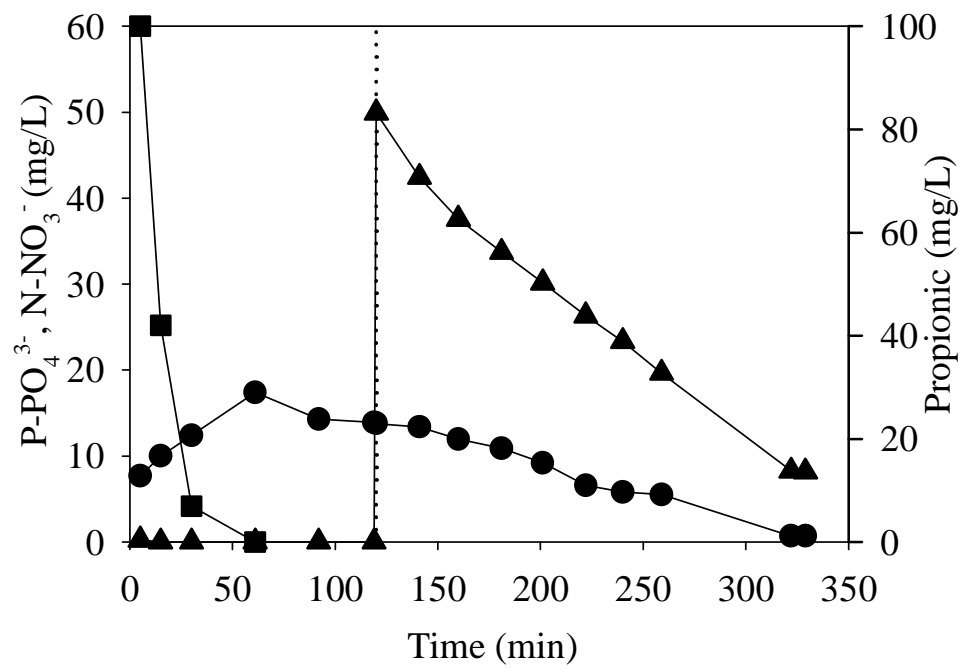

566 Figure S2 Experimental propionic ( $\mathbf{-}), \mathrm{P}(\bullet)$ and $\mathrm{NO}_{3}{ }^{-}(\mathbf{\Delta})$ profiles for a cycle at day 56730.

568

569 